\title{
Uniqueness in Determining Polygonal Periodic Structures
}

\author{
J. Elschner and M. Yamamoto
}

\begin{abstract}
We consider the inverse problem of recovering a two-dimensional perfectly reflecting diffraction grating from scattered waves measured above the structure. We establish the uniqueness within the class of general polygonal grating profiles by a minimal number of incoming plane waves, without excluding Rayleigh frequencies and further geometric constraints on the profile. This extends and improves the uniqueness results of Elschner, Schmidt and Yamamoto [Inverse Problems 19 (2003), 779-787].
\end{abstract}

Keywords. Diffraction grating, periodic Helmholtz equation, inverse Dirichlet and Neumann problems, polygonal grating profile

Mathematics Subject Classification (2000). Primary 78A46, secondary 35R30

\section{Introduction}

The problem of recovering a periodic structure from knowledge of the scattered field occurs in many applications, e.g., in diffractive optics; see $[3,18]$. In this paper we consider the scattering of monochromatic plane waves by a perfectly reflecting diffraction grating in an isotropic lossless medium. Our goal is to prove global uniqueness in determining polygonal periodic grating profiles by near field observations with a minimal number of incident waves.

Let the profile of the diffraction grating be given by a Lipschitz curve $\Lambda \subset \mathbb{R}^{2}$ which is $2 \pi$-periodic with respect to $x_{1}$, where $\left(x_{1}, x_{2}\right) \in \mathbb{R}^{2}$. The unbounded domain above $\Lambda$ is denoted by $\Omega_{\Lambda}$. Suppose that a plane wave given by

$$
u^{i}(x):=\exp \left(i \alpha x_{1}-i \beta x_{2}\right), \quad(\alpha, \beta)=k(\sin \theta, \cos \theta)
$$

is incident from the top, where $k>0$ is the wave number and $\theta \in\left(-\frac{\pi}{2}, \frac{\pi}{2}\right)$ is the incident angle.

J. Elschner: Weierstrass Institute for Applied Analysis and Stochastics, Mohrenstr. 39, 10117 Berlin, Germany; elschner@wias-berlin.de

M. Yamamoto: Graduate School of Mathematical Sciences, University of Tokyo, 3-8-1 Komaba Meguro, Tokyo 153-8914, Japan; myama@ms.u-tokyo.ac.jp 
We consider the scattering of $u^{i}$ in the case of a perfectly reflecting grating profile $\Lambda$, which is modeled by the Dirichlet problem (TE polarization) or the Neumann problem (TM polarization). Then the total field $u=u\left(x_{1}, x_{2}\right)$, which is the sum of $u^{i}$ and the scattered field $u^{s}$, satisfies

$$
\Delta u+k^{2} u=0 \quad \text { in } \Omega_{\Lambda}, \quad u=0 \quad \text { or } \quad \partial_{\nu} u=0 \quad \text { on } \Lambda,
$$

and is assumed to be $\alpha$-quasiperiodic in $x_{1}$ :

$$
u\left(x_{1}+2 \pi, x_{2}\right)=\exp (2 i \alpha \pi) u\left(x_{1}, x_{2}\right) .
$$

Furthermore, $u^{s}$ is required to satisfy the radiation condition

$$
u^{s}(x)=\sum_{n \in \mathbb{Z}} A_{n} \exp \left(i(n+\alpha) x_{1}+i \beta_{n} x_{2}\right) \quad \text { for } x_{2} \text { sufficiently large, }
$$

with the Rayleigh coefficients $A_{n} \in \mathbb{C}$ and

$$
\beta_{n}:= \begin{cases}\left(k^{2}-(n+\alpha)^{2}\right)^{\frac{1}{2}}, & \text { if }|n+\alpha| \leq k \\ i\left((n+\alpha)^{2}-k^{2}\right)^{\frac{1}{2}}, & \text { if }|n+\alpha|>k .\end{cases}
$$

Note that $\beta_{n}$ is real for at most a finite number of indices.

There always exists a solution $u \in H_{l o c}^{1}\left(\Omega_{\Lambda}\right)$ of problem (1)-(3) which need not be unique in general. For the existence result see, e.g., $[5,7]$ where the more general TE and TM transmission problems have been studied, and nonuniqueness examples for both the direct Dirichlet and Neumann problems can be found in [13]. Moreover, it is known that the solution to the Dirichlet problem is always unique if the profile curve $\Lambda$ is given by the graph of a function (see [16] for $C^{2}$ and [8] for Lipschitz functions), whereas this may be not true for the Neumann problem [15].

The inverse Dirichlet or Neumann problem can now be formulated as follows:

(IP): Determine the profile $\Lambda$ from the knowledge of one wave number $k$, possibly several incident directions $\theta$, and the total field $\left.u\right|_{x_{2}=b}$ on a straight line $\left\{x \in \mathbb{R}^{2}: x_{2}=b\right\}$ lying in $\Omega_{\Lambda}$.

Note that this problem also involves near field measurements since the evanescent modes cannot be measured far away from the grating profile.

In general, global uniqueness with one incident wave in problem (IP) is not true. This can be seen from the simple counterexample of the scattering of $u^{i}=\exp \left(-i k x_{2}\right)$ when one moves the flat grating in certain multiples of the wavelength. It was shown in [14] that a finite number of incident waves are sufficient to recover a $C^{2}$ grating profile from the total field above the structure. 
In particular, one obtains the global uniqueness with one incident direction in the inverse Dirichlet problem if the wave number or the amplitude of the grating is sufficiently small.

Global uniqueness results for the inverse Dirichlet and Neumann problems with a minimal number of incident waves were first established within the class of profiles given by the graph of a piecewise linear function or a step function $[10,11]$. The purpose of this paper is to extend these results to the practically important case of general polygonal grating profiles. Moreover, contrary to [10], we can allow Rayleigh frequencies and are able to remove the additional geometric assumption on the profile made in the Neumann case. Now we state our main result.

Theorem. Let $\Lambda_{1}$ and $\Lambda_{2}$ be $2 \pi$-periodic polygonal profiles consisting of finitely many segments, and let us exclude the case where $\Lambda_{1}$ and $\Lambda_{2}$ are parallels to the $x_{1}$ axis. Let $u_{j}=u\left(\Lambda_{j} ; \theta\right)$ satisfy the corresponding direct diffraction problem (1)-(3) in $\Omega_{j}=\Omega_{\Lambda_{j}}, j=1,2$, and choose $b$ such that $\left\{x \in \mathbb{R}^{2}: x_{2}=b\right\} \subset$ $\Omega_{1} \cap \Omega_{2}$.

(i) In the Dirichlet case the relations

$$
u_{1}\left(x_{1}, b\right)=u_{2}\left(x_{1}, b\right) \quad \text { for all } x_{1} \in(0,2 \pi)
$$

for two different incident angles $\theta$ imply $\Lambda_{1}=\Lambda_{2}$. If one excludes the Rayleigh frequencies by assuming

$$
\beta_{n} \neq 0, \quad \text { i.e. }, \quad k^{2} \neq(n+\alpha)^{2} \quad \text { for all } n \in \mathbb{Z},
$$

then the relation (5) for one incident wave is sufficient.

(ii) For the inverse Neumann problem, we have $\Lambda_{1}=\Lambda_{2}$ if the relations (5) hold for four different incident directions, whereas three incoming waves are enough if the Rayleigh frequencies are excluded for each incident angle $\theta$.

It will be shown by appropriate counterexamples that a smaller number of incident waves is not sufficient to determine the grating profile uniquely, in general. We refer to the remarks at the end of the paper.

The proof of the theorem is carried out in the next section and relies on a refinement of the arguments in [10] in combination with those developed in $[1,6,9]$ for inverse scattering by polygonal sound-soft and sound-hard obstacles.

\section{Proof of the theorem}

2.1. Finding an "exit direction". Arguing by contradiction, let $\Lambda_{1} \neq \Lambda_{2}$ be two periodic polygonal curves, with the case $\Lambda_{j}=\left\{x \in \mathbb{R}^{2}: x_{2}=c_{j}\right\}, c_{1}<c_{2}$, excluded. Consider the solutions $u_{j}=u\left(\Lambda_{j} ; \theta\right) \in H_{l o c}^{1}\left(\Omega_{j}\right)$ of the corresponding 
direct diffraction problems, and let $\Omega$ be the unbounded connected component of $\Omega_{1} \cap \Omega_{2}$. Note that by elliptic regularity, each function $u_{j}$ is infinitely smooth up to the boundary, with the exception of the corner points of $\Lambda_{j}$. Moreover, since $u_{j}$ satisfies the Helmholtz equation, $u_{j}$ is real-analytic in $\Omega_{j}$. Since $u_{1}=u_{2}$ and then also $\partial_{2} u_{1}=\partial_{2} u_{2}$ on $\left\{x_{2}=b\right\}$, we have

$$
u(x)=u(\theta)(x):=u_{1}(x)=u_{2}(x) \text { in } \Omega ;
$$

see, e.g., [2]. Henceforth by a ray we mean a straight line starting from one point and extended to infinity.

The following auxiliary result extends Lemma 2 in [10] to general polygonal grating profiles. Note that the proof is more straightforward in the case where $\Lambda_{1}$ and $\Lambda_{2}$ are given by the graphs of piecewise linear functions; see [10, Sec. 2.2].

Proposition 1. There exists a ray $S \subset \Omega$ such that $\left.u\right|_{S}=0$ in the Dirichlet case and $\left.\partial_{\nu} u\right|_{S}=0$ in the Neumann case.

Proof. Henceforth $A_{1} A_{2}$ stands for the open segment in $\mathbb{R}^{2}$ with end points $A_{1}$ and $A_{2}$. Since $\Lambda_{1} \neq \Lambda_{2}$, there exists a segment $A_{1} A_{2} \subset \partial \Omega \cap \Omega_{1}$ without loss of generality (see [9, Sec. 3] for the details), and by (7) we have $u_{1}=0$ resp. $\partial_{\nu} u_{1}=0$ on $A_{1} A_{2}$. Thus the set $\mathcal{G}$ defined by

$\mathcal{G}=\left\{S: \begin{array}{l}S \text { is a (finite or infinite) open segment extended to maximal length } \\ \text { in } \Omega_{1} \text { such that } u_{1}=0 \text { resp. } \partial_{\nu} u_{1}=0 \text { on } S\end{array}\right\}$

is not empty. If $\mathcal{G}$ contains an infinite segment, then we already have the desired ray. So we may assume that the subset of finite segments is not empty, and by periodicity we can restrict ourselves to the set

$\mathcal{G}_{0}=\left\{\begin{array}{l}S \text { is a finite open segment with both ends on } \Lambda_{1} \text {, starting in a fixed } \\ S: \text { period, say } 0 \leq x_{1} \leq 2 \pi, \text { and ending in the same, in the preceding } \\ \text { or subsequent period, such that } u_{1}=0 \text { resp. } \partial_{\nu} u_{1}=0 \text { on } S\end{array}\right\}$.

Proceeding similarly to the case of scattering by polygonal bounded obstacles (cf. $[1,6,9]$ ), we can prove that $\mathcal{G}_{0}$ is a finite set, which then implies the existence of a ray $S$ with the desired properties or a contradiction to the assumption that $\Lambda_{1} \neq \Lambda_{2}$. Let us give a sketch of the finiteness proof, first for the inverse Dirichlet problem and then for the Neumann case, and we refer to $[6,9]$ for the details.

If $\mathcal{G}_{0}$ contains infinitely many segments, we can choose sequences of points $\left\{P_{j}\right\},\left\{Q_{j}\right\}$ such that $P_{j} \neq P_{j^{\prime}}$ if $j \neq j^{\prime}, P_{j}, Q_{j} \in \Lambda_{1}, P_{j} Q_{j} \subset \Omega_{1}$ and $u_{1}=0$ on $P_{j} Q_{j}$ for all $j$. Since the length of $\Lambda_{1}$ restricted to $\left\{x \in \mathbb{R}^{2}:-2 \pi \leq x_{1} \leq 4 \pi\right\}$ is finite, we find subsequences $P_{j} \rightarrow P_{\infty}, Q_{j} \rightarrow Q_{\infty}$, lying at one side of $P_{\infty}, Q_{\infty}$, respectively. Moreover, $P_{j}$ are not vertices of $\Lambda_{1}$ and $P_{j} P_{j+1}, Q_{j} Q_{j+1} \subset \Lambda_{1}$ 
for all $j$. It may happen that the stationary sequence $Q_{j}=Q_{\infty}$ occurs or that $P_{\infty}=Q_{\infty}$. Consider the polygonal open set $D_{j}$ bounded by the segments $P_{j} Q_{j}, P_{j} P_{j+1}, P_{j+1} Q_{j+1}, Q_{j} Q_{j+1}$, which is a triangle or quadrangle, or consists of two triangles with a common vertex.

For any $j, u_{1}$ satisfies the homogeneous Dirichlet problem for the Helmholtz operator $\Delta+k^{2}$ on $D_{j}$, and the area of $D_{j}$ tends to zero as $j \rightarrow \infty$. However, this contradicts the Poincaré inequality for $H^{1}$ functions with vanishing boundary values (see, e.g., [12, Ch. 7.8]) and thus proves the finiteness of the set $\mathcal{G}_{0}$.

In the case of the Neumann problem, more sophisticated arguments are needed to show that the corresponding set $\mathcal{G}_{0}$ is finite. As in [6] or [9, Sec. 3], we can construct a sequence of triangles $D_{j}$, whose diameters converge to zero as $j \rightarrow \infty$, and such that $u_{1}$ satisfies the homogeneous Neumann problem for the Helmholtz operator on $D_{j}$ for all $j$. Then we obtain a contradiction to the optimal Poincaré inequality of [17] for planar convex domains. In the $n$-dimensional case when $n \geq 3$, a gap in the original proof of this inequality was indicated and fixed in [4].

We now complete the proof of Proposition 1 following the proof of Lemma 3.7 in [1]. Consider the open set

$$
G^{*}:=\left(\Omega_{1} \backslash \bigcup_{S \in \mathcal{G}_{0}} S\right) \cap\left\{x \in \mathbb{R}^{2}: 0<x_{1}<2 \pi\right\}
$$

which has one unbounded connected component $G_{\infty}$ and a finite number of bounded connected components. We note that there exists only one unbounded connected component, because the boundaries of any components of $\Omega_{1} \backslash \mathcal{G}_{0}$ consist of finite segments.

Now we choose a point $P \in \partial G_{\infty}$ lying on exactly one open segment $S$ of $\mathcal{G}_{0}$, and select points $P^{+} \in G_{\infty}$ and $P^{-} \in G^{*} \backslash \bar{G}_{\infty}$ sufficiently close to $P$. Moreover, we can choose a continuous curve $\gamma(t), t \in\left[t_{1}, \infty\right)$, which intersects the set $\mathcal{G}_{0}$ only at the point $P \in S$ and satisfies, for some $t_{2}>\bar{t}>t_{1}$,

$$
\begin{gathered}
\gamma\left(t_{1}\right)=P^{-} ; \quad \gamma(t) \in G^{*} \backslash \bar{G}_{\infty}, \quad t \in\left(t_{1}, \bar{t}\right) \\
\gamma(\bar{t})=P ; \quad \gamma(t) \in G_{\infty}, \quad t>\bar{t} \\
\text { with } \gamma\left(t_{2}\right)=P^{+} \text {and } \gamma(t) \text { leading to infinity as } t \rightarrow \infty .
\end{gathered}
$$

We will find a segment $S^{*} \neq S$ with $u_{1}=0$ on $S^{*}$, intersecting $\gamma$ at some $t>\bar{t}$. Then, either $S^{*}$ can be extended to a ray in $\Omega$ on which $u_{1}$ vanishes (an exit direction), or we have a finite segment belonging to $\mathcal{G}_{0}$ which is a contradiction.

Let $G^{ \pm}$be the connected components of $\left(\Omega_{1} \backslash S\right) \cap\left\{x \in \mathbb{R}^{2}: 0<x_{1}<2 \pi\right\}$ containing $P^{ \pm}$, and consider the connected components $E^{ \pm}$of $G^{ \pm} \cap \Pi\left(G^{\mp}\right)$ containing $P^{ \pm}$, where $\Pi$ denotes the symmetric transform with respect to the 
extended straight line of $S$. Then $E^{-}=\Pi\left(E^{+}\right)$. Let $E=E^{-} \cup E^{+} \cup S$. Note that $\partial E$ consists of segments of $\Lambda_{1}$ and $\Pi\left(\Lambda_{1}\right)$.

Since $u_{1}$ is odd symmetric with respect to $S$, we obtain $u_{1}=0$ on $\partial E$. Note that $E$ is bounded since $G^{-}$is bounded. Therefore, for some $t>\bar{t}, \gamma(t)$ intersects $\partial E \cap G_{\infty}$ and thus another segment $S^{*} \neq S$ with $u_{1}=0$ on $S^{*}$. This finishes the proof of Proposition 1 in the Dirichlet case.

The proof in the Neumann case is analogous; we only have to use the fact that $u_{1}$ is even symmetric with respect to $S$ in the above argument.

2.2. Reduction to a finite sum of propagating waves. The function $u$ defined in (7) has the Rayleigh expansion

$$
\begin{aligned}
u(x)= & u(\theta)(x) \\
= & \left(A \exp \left(i \alpha x_{1}-i \beta x_{2}\right)+\sum_{n \in P} A_{n} \exp \left(i(\alpha+n) x_{1}+i \beta_{n} x_{2}\right)\right) \\
& +\left(\sum_{n \in \mathbb{Z} \backslash P} A_{n} \exp \left(i(n+\alpha) x_{1}+i \beta_{n} x_{2}\right)\right):=v+w \quad\left(x_{2}>b\right),
\end{aligned}
$$

where $A=1$ and $P$ denotes the finite set $\left\{n \in \mathbb{Z}: \beta_{n} \in \mathbb{R}\right\}$. Note that $-i \beta_{n} \geq C>0$ for all $n \in \mathbb{Z} \backslash P$ and $\beta_{n} \sim|n| i$ as $|n| \rightarrow \infty$. Of course, $\alpha, \beta, \beta_{n}$ and $A_{n}$ depend on the incident direction $\theta$.

Proposition 2. The existence of an exit direction (cf. Proposition 1) implies that $w=0$ in (8), i.e., $A_{n}=0$ for all $n \in \mathbb{Z} \backslash P$.

Proof. Since a translation of the $x$ coordinates only amounts to different coefficients in the expansion (8) with $A \neq 0$, we can assume that the ray $S$ of Proposition 1 starts at $x=0$. We have

$$
\partial_{\nu} u=-\sin \phi \partial_{1} u+\cos \phi \partial_{2} u \quad \text { on } S
$$

if the ray $S$ is given by $S=\{(t \cos \phi, t \sin \phi): t>0\}$. We have to consider the following two cases:

(i) Dirichlet case: As in [10], $\left.u\right|_{S}=0$ implies $A_{n}=0$ for all $n \in \mathbb{Z} \backslash P$ if $\phi \neq 0$. For $\phi=0$, we obviously even have $A_{n}=0$ for $n \neq 0$.

(ii) Neumann case: From (8) and (9) we have

$$
\begin{aligned}
\partial_{\nu} u(x) & =B \exp \left(i \alpha x_{1}-i \beta x_{2}\right)+\sum_{n \in \mathbb{Z}} B_{n} \exp \left(i(\alpha+n) x_{1}+i \beta_{n} x_{2}\right) \quad \text { on } S \\
B & =(-i \alpha \sin \phi-i \beta \cos \phi) A \\
B_{n} & =\left(-i(n+\alpha) \sin \phi+i \beta_{n} \cos \phi\right) A_{n}, \quad n \in \mathbb{Z} .
\end{aligned}
$$


Then, for $n \in \mathbb{Z} \backslash P$, we have $B_{n} \neq 0$ if and only if $A_{n} \neq 0$. Note that $\beta_{n} \notin \mathbb{R}$ for these $n$, implying the assertion for $\phi \neq \frac{\pi}{2}$, and that by (4) $|n+\alpha|>k>0$ for $n \in \mathbb{Z} \backslash P$ if $\phi=\frac{\pi}{2}$. Then Proposition 2 follows as in the Dirichlet case; see $[10]$.

2.3. End of proof in the Dirichlet case. We now have from (8) and Proposition 2 that

$$
u=v=A \exp \left(i \alpha x_{1}-i \beta x_{2}\right)+\sum_{n \in P} A_{n} \exp \left(i(\alpha+n) x_{1}+i \beta_{n} x_{2}\right), A \neq 0 .
$$

Moreover, $v$ is analytic in $\mathbb{R}^{2}$ and satisfies $\left.v\right|_{L}=0$ on each straight line $L$ extending a segment of $\Lambda_{1} \cup \Lambda_{2}$. So, by assumption, there exist at least two lines $L_{1}, L_{2}$ intersecting at $x=0$, where $v$ vanishes. Let $\psi$ denote the polar angle between $L_{1}$ and $L_{2}$. Using the reflection argument with odd extension, we end up with the following two cases (cf. [10]):

(i): $\psi=\lambda \pi, \lambda \in(0,1)$ irrational, and $\left.v\right|_{L_{j \psi}}=0$ for all $j \in \mathbb{N}$.

Here $L_{j \psi}$ denotes the line of polar angle $j \psi$ with respect to $L_{1}$. Since the directions of $L_{j \psi}$ are dense in $[0,2 \pi)$, we obtain $v=0$ in $\mathbb{R}^{2}$ which is a contradiction to $A \neq 0$. Therefore, case (i) cannot occur.

(ii): $\psi=\lambda \pi, \lambda$ rational, and $\left.v\right|_{L_{j \pi / N}}=0, j=0, \ldots, N-1$, for some integer $N \geq 2$.

Since $v$ is odd symmetric with respect to the lines $L_{j \pi / N}$, we obtain $v(x)=$ $(-1)^{N} v(-x), \quad x \in \mathbb{R}^{2}$, or equivalently,

$$
\begin{aligned}
& A \exp \left(i \alpha x_{1}-i \beta x_{2}\right)+\sum_{n \in P} A_{n} \exp \left(i(n+\alpha) x_{1}+i \beta_{n} x_{2}\right) \\
& =(-1)^{N} A \exp \left(-i \alpha x_{1}+i \beta x_{2}\right)+(-1)^{N} \sum_{n \in P} A_{n} \exp \left(-i(n+\alpha) x_{1}-i \beta_{n} x_{2}\right) ;
\end{aligned}
$$

see [10]. This implies, for some $n_{0} \in P$ and all $x \in \mathbb{R}^{2}$,

$$
(-1)^{N} A \exp \left(-i \alpha x_{1}+i \beta x_{2}\right)=A_{n_{0}} \exp \left(i\left(n_{0}+\alpha\right) x_{1}+i \beta_{n_{0}} x_{2}\right),
$$

and because of $A \neq 0$,

$$
n_{0}=-2 \alpha, \quad \beta_{n_{0}}=\beta, \quad A(-1)^{N}=A_{n_{0}} .
$$

Moreover, (12) gives for all $x \in \mathbb{R}^{2}$

$$
\begin{aligned}
& \sum_{n \in P \backslash\left\{n_{0}\right\}} A_{n} \exp (\left.i(n+\alpha) x_{1}+i \beta_{n} x_{2}\right) \\
&=(-1)^{N} \sum_{n \in P \backslash\left\{n_{0}\right\}} A_{n} \exp \left(-i(n+\alpha) x_{1}-i \beta_{n} x_{2}\right) .
\end{aligned}
$$


Therefore, for any $n \in P \backslash\left\{n_{0}\right\}$, we must have either $A_{n}=0$, or $\beta_{n}=0$ in which case a Rayleigh frequency occurs. If there is no Rayleigh frequency, we have $A_{n}=0$ for all $n \in P \backslash\left\{n_{0}\right\}$. Otherwise there exists $n_{1} \in \mathbb{Z}$ with $\beta_{n_{1}}=0$ and $n_{1}+\alpha=k$, and by equality (14) there is another index $n_{2} \in \mathbb{Z}$ such that

$$
\beta_{n_{1}}=\beta_{n_{2}}=0, \quad n_{1}+\alpha=-\left(n_{2}+\alpha\right)=k, \quad A_{n_{2}}=(-1)^{N} A_{n_{1}} .
$$

Furthermore, we have $A_{n}=0$ if $n \neq n_{0}, n_{1}, n_{2}$. Hence, in terms of (11), (13) and (15), for even $N, v$ takes the form

$$
v(x)=A \cos \left(\alpha x_{1}-\beta x_{2}\right)+B \cos \left(k x_{1}\right), \quad A \neq 0,
$$

where $B=0$ if there is no Rayleigh frequency. Similarly, for odd $N$, cos has to be replaced by sin:

$$
v(x)=A \sin \left(\alpha x_{1}-\beta x_{2}\right)+B \sin \left(k x_{1}\right), \quad A \neq 0 .
$$

To complete the proof of the theorem in the Dirichlet case, we now investigate the relation $\left.v\right|_{L}=0$, where $v$ is given by (16) or (17), and $L$ is the straight line given by $L=L(\phi)=\{(t \cos \phi, t \sin \phi): t \in \mathbb{R}\}$, where $\phi \in\left(-\frac{\pi}{2}, \frac{\pi}{2}\right]$. Since $(\alpha, \beta)=k(\sin \theta, \cos \theta)$ with $\theta \in\left(-\frac{\pi}{2}, \frac{\pi}{2}\right)$, we have $\alpha x_{1}-\beta x_{2}=t k \sin (\theta-\phi)$ on $L(\phi)$, and (16) can be written as

$$
A \cos (t k \sin (\theta-\phi))+B \cos (t k \cos \phi)=0, \quad t \in \mathbb{R} .
$$

This is impossible for $B=0$. To explore the case $B \neq 0$, we note that for $a, b \in \mathbb{R}$ the functions

$$
\cos (a t), \cos (b t) \text { are linearly independent on } \mathbb{R} \text { if and only if } a= \pm b .
$$

Hence (18) implies that

$$
\sin (\theta-\phi)= \pm \cos \phi= \pm \sin \left(\frac{\pi}{2}-\phi\right)
$$

giving the relations $\theta-\phi=\frac{\pi}{2}+\phi$ or $\theta-\phi=\phi-\frac{\pi}{2}$ under the above constraints on $\theta$ and $\phi$.

Therefore, the functions (16) can only vanish on the line $L(\phi)$ if, for the given incident direction $\theta$,

$$
\phi=\frac{\theta}{2}-\frac{\pi}{4} \quad \text { or } \quad \phi=\frac{\theta}{2}+\frac{\pi}{4} .
$$

On the other hand, we know that $v$ vanishes on two lines $L_{j}=L\left(\phi_{j}\right), j=1,2$, where $\phi_{1}<\phi_{2}$ without loss of generality. Hence, by (21) we have

$$
\theta=2 \phi_{1}+\frac{\pi}{2}=2 \phi_{2}-\frac{\pi}{2} .
$$


Now we observe that the relations (22) are not possible for another incident direction $\theta_{1} \neq \theta$. This completes the proof if $v$ has the representation (16).

Finally we consider the case $\left.v\right|_{L}=0$ with $L=L(\phi)$ and $v$ given by (17). Then we have

$$
A \sin (t k \sin (\theta-\phi))+B \sin (t k \cos \phi)=0, \quad t \in \mathbb{R} .
$$

For $B=0,(23)$ implies that $\phi=\theta$ which is not possible for $\phi=\phi_{1}, \phi_{2}, \phi_{1}<\phi_{2}$. Thus we have proved the uniqueness with one incident wave if the Rayleigh frequencies are excluded.

We are left with the case $B \neq 0$, i.e., there exist Rayleigh frequencies. Since (19) also holds with cos replaced by sin, we obtain the relations (20)-(22) again. However, taking a different incident direction $\theta_{1} \neq \theta$, we obtain a contradiction as above. This finishes the proof of assertion (i) of the theorem.

2.4. End of proof in the Neumann case. This time the finite expansion (11) satisfies $\left.\partial_{\nu} u\right|_{L}=0$ on each straight line extending a segment of $\Lambda_{1} \cup \Lambda_{2}$. By assumption, there are at least two lines $L_{j}=L\left(\phi_{j}\right), j=1,2, \phi_{1}<\phi_{2}$, with this property. Let again $\psi=\phi_{2}-\phi_{1}$ be the angle between $L_{1}$ and $L_{2}$. Using now the reflection argument with even extension, we arrive at the following two cases (compare Subsection 2.3):

(i): $\psi=\lambda \pi, \lambda \in(0,1)$ irrational, and $\left.\partial_{\nu} v\right|_{L_{j \psi}}=0$ for all $j \in \mathbb{N}$.

Hence we have $\left.\partial_{\nu} v\right|_{L}=0$ for all directions $L=L(\phi), \phi \in[0,2 \pi)$. Passing to polar coordinates $x=r(\cos \phi, \sin \phi)$ and multiplying by $r$, from (10) we obtain the relation

$$
\begin{aligned}
-\left(i \alpha x_{2}\right. & \left.+i \beta x_{1}\right) A \exp \left(i \alpha x_{1}-i \beta x_{2}\right) \\
& +\sum_{n \in P}\left(-i(n+\alpha) x_{2}+i \beta_{n} x_{1}\right) A_{n} \exp \left(i(n+\alpha) x_{1}+i \beta_{n} x_{2}\right)=0
\end{aligned}
$$

for all $x \in \mathbb{R}^{2}$. However, (24) is not possible since $A \neq 0$ and the functions on the left hand side of this equality are linearly independent on $\mathbb{R}^{2}$. Therefore the case (i) cannot happen.

(ii): $\lambda$ rational, and $\left.\partial_{\nu} v\right|_{L_{j \pi / N}}=0, j=0, \ldots, N-1$, for some integer $N \geq 2$. Since $v$ is symmetric with respect to the lines $L_{j \pi / N}$, we obtain this time $v(x)=$ $v(-x), x \in \mathbb{R}^{2}$, implying the relations (12)-(15) without the factor $(-1)^{N}$. Therefore, $v$ takes the form (16), and we have to investigate the relation $\left.\partial_{\nu} v\right|_{L}=$ 0 with $L=L(\phi)$, which can be written as

$$
\left.\partial_{\nu} v\right|_{L}=(\alpha \sin \phi+\beta \cos \phi) A \sin (t k \sin (\theta-\phi))+B k \sin \phi \sin (t k \cos \phi)=0
$$

for $t \in \mathbb{R}$, or equivalently,

$$
A \cos (\theta-\phi) \sin (t k \sin (\theta-\phi))+B \sin \phi \sin (t k \cos \phi)=0, \quad t \in \mathbb{R} .
$$


The last relation holds for $\phi=\phi_{j}, j=1,2, \phi_{1}<\phi_{2}$. First we consider the case that $\phi_{1}=0$ or $\phi_{2}=\frac{\pi}{2}$. Then (25) and $\theta \in\left(-\frac{\pi}{2}, \frac{\pi}{2}\right)$ imply that $\theta=0$, so that two incident directions are enough to determine the profile in this case.

From now on, we can assume that $\phi_{1}, \phi_{2} \notin\left\{0, \frac{\pi}{2}\right\}$. If $B=0$ (e.g., no Rayleigh frequency occurs), then (25) gives

$$
\theta=\phi, \quad \text { or } \quad \theta=\phi-\frac{\pi}{2} \quad \text { if } \phi>0 \quad \text { and } \quad \theta=\phi+\frac{\pi}{2} \quad \text { if } \phi<0 .
$$

Hence, by $\phi_{1}, \phi_{2} \in\left(-\frac{\pi}{2}, \frac{\pi}{2}\right) \backslash\{0\}$, we have $\phi_{1}<0<\phi_{2}$, so that

$$
\phi_{1}=\theta \quad \text { or } \quad \phi_{2}=\theta, \quad \text { with } \phi_{2}-\phi_{1}=\frac{\pi}{2},-\frac{\pi}{2}<\phi_{1}<0<\phi_{2}<\frac{\pi}{2} \text {. }
$$

From (26) we observe that three different incident directions $\theta$ with $B=B(\theta)=0$ in (25) and $\left.\partial_{\nu} v(\theta)\right|_{L_{j}}=0, j=1,2$, are impossible. This proves assertion (ii) of the theorem if Rayleigh frequencies are excluded for each incoming wave.

Finally, let $\theta$ be an incident direction such that (15) holds and (25) is fulfilled with $B \neq 0$ and $\phi=\phi_{j}, j=1,2, \phi_{1}<\phi_{2}$. Since $B \sin \phi \sin (t k \cos \phi)$ does not vanish identically in $\mathbb{R}$ by $\phi \in\left(-\frac{\pi}{2}, \frac{\pi}{2}\right) \backslash\{0\}$, we have $\cos (\theta-\phi) \neq 0$ in (25), and as in Subsection 2.3 we further obtain relations (22). Note that these relations cannot be true for another incident direction $\theta_{1} \neq \theta$ unless the corresponding coefficient $B=B\left(\theta_{1}\right)$ in $(25)$ is zero. Since the equality $B(\theta)=0$ can only hold for at most two different incident angles $\theta$, we have thus proved that measurements with four incoming waves are always sufficient to ensure the uniqueness in the inverse Neumann problem. This finishes the proof of the theorem.

Remark 1. The counterexamples of [10] show that in the case of Rayleigh frequencies one incident wave is not enough to ensure the uniqueness in the inverse Dirichlet problem in general.

We now present an example for non-uniqueness in the inverse Neumann problem with two incident waves if no Rayleigh frequencies occur:

Consider the quadratic grid generated by the $2 \pi$-periodic extensions of the lines $L(\phi), \phi= \pm \frac{\pi}{4}$, and the incident waves with $\theta_{1}=-\frac{\pi}{4}, \theta_{2}=\frac{\pi}{4}$, and let $k=\frac{1}{\sqrt{2}}$. Then we have

$$
\begin{aligned}
& k \sin \theta_{j}=\frac{(-1)^{j}}{2}, \quad k \cos \theta_{j}=\frac{1}{2}, \\
& k^{2}=\frac{1}{2} \neq\left(n+k \sin \theta_{j}\right)^{2}=n^{2}+(-1)^{j} n+\frac{1}{4} \quad \text { for all } n \in \mathbb{Z},
\end{aligned}
$$

i.e., no Rayleigh frequencies for $\theta_{1}$ and $\theta_{2}$, and the functions

$$
v_{j}(x)=v\left(\theta_{j}\right)(x)=2 \cos \left((-1)^{j} \frac{x_{1}}{2}-\frac{x_{2}}{2}\right)
$$


satisfy the Helmholtz equation in the whole plane and the corresponding quasiperiodicity and radiation conditions. Note that (11) takes the form (16) with $A=2$ and $B=0$ for $\theta=\theta_{1}, \theta_{2}$. Moreover, the normal derivatives $\partial_{\nu} v_{j}, j=1,2$, vanish on the lines $\left\{x_{2}= \pm x_{1}\right\}$ since

$$
\left.\partial_{\nu} v_{j}\right|_{L\left( \pm \frac{\pi}{4}\right)}=2 \cos \left(\theta_{j} \pm \frac{\pi}{4}\right) \sin \left(t k \sin \left(\theta_{j} \pm \frac{\pi}{4}\right)\right)=0
$$

for all $t \in \mathbb{R}$ and $j=1,2$; compare the left hand side of (25).

Remark 2. To construct a non-uniqueness example for the inverse Neumann problem with three incident angles $\theta_{j}, j=1,2,3$, we consider a rectangular grid generated by the $2 \pi$-periodic extensions of two lines $L_{i}=L\left(\phi_{i}\right), i=1,2$, $\phi_{1}<\phi_{2}$. We have to show that, for each $j$, there is a solution $v_{j}=v\left(\theta_{j}\right)$ of the Helmholtz equation in the whole plane satisfying homogeneous Neumann conditions on both lines $L_{i}$ as well as the corresponding quasiperiodicity and radiation conditions. Then, by the considerations at the end of the above proof, we are left with the following case:

$$
\phi_{1}=\theta_{2}=\frac{\theta_{1}}{2}-\frac{\pi}{4}<0, \quad \phi_{2}=\theta_{3}=\frac{\theta_{1}}{2}+\frac{\pi}{4}>0 ;
$$

compare (22) and (26). Moreover, each $v\left(\theta_{j}\right)$ takes the form (16) with $B\left(\theta_{1}\right) \neq 0$, $B\left(\theta_{2}\right)=B\left(\theta_{3}\right)=0$, and a Rayleigh frequency occurs at least for $\theta=\theta_{1}$.

Let $k=\frac{25}{2}$, and choose the incident angles $\theta_{j}$ such that

$$
\sin \theta_{1}=\frac{7}{25}, \quad \sin \theta_{2}=-\frac{3}{5}, \quad \sin \theta_{3}=\frac{4}{5}
$$

implying the relations

$$
\begin{aligned}
& \cos \theta_{2}=\sin \theta_{3}=\frac{4}{5}, \quad \cos \theta_{3}=-\sin \theta_{2}=\frac{3}{5}, \\
& \cos \theta_{1}=\frac{24}{25}=\cos \left(2 \theta_{2}+\frac{\pi}{2}\right)=-\sin 2 \theta_{2} .
\end{aligned}
$$

Therefore, the incident angles satisfy the constraints (27), and from (28) and (29) we further have that

$$
\begin{aligned}
& k\left(\sin \theta_{1}, \cos \theta_{1}\right)=\left(\frac{7}{2}, 12\right), \quad k\left(\sin \theta_{2}, \cos \theta_{2}\right)=\left(-\frac{15}{2}, 10\right), \\
& k\left(\sin \theta_{3}, \cos \theta_{3}\right)=\left(10, \frac{15}{2}\right)
\end{aligned}
$$

and the straight lines $L_{i}$ are given by

$$
L_{1}=\left\{\left(\frac{4}{5} t,-\frac{3}{5} t\right): t \in \mathbb{R}\right\}, \quad L_{2}=\left\{\left(\frac{3}{5} t, \frac{4}{5} t\right): t \in \mathbb{R}\right\} .
$$


Using the ansatz (16) (with $A=2$ for each incident direction), together with (30) and (31), it can easily be checked that the functions $v_{j}$ defined by

$$
\begin{aligned}
& v_{1}(x)=2 \cos \left(\frac{7}{2} x_{1}-12 x_{2}\right)+2 \cos \left(\frac{25}{2} x_{1}\right) \\
& v_{2}(x)=2 \cos \left(\frac{15}{2} x_{1}+10 x_{2}\right), \quad v_{3}(x)=2 \cos \left(10 x_{1}-\frac{15}{2} x_{2}\right)
\end{aligned}
$$

are the required solutions to the Helmholtz equation. In particular, the functions (32) satisfy homogeneous Neumann conditions on $L_{1} \cup L_{2}$ and thus on the grid generated by the $2 \pi$-periodic extensions of these lines. Moreover, for $\theta=\theta_{1}$, we note that (15) holds with $n_{1}=9$ and $n_{2}=-16$, so that indeed a Rayleigh frequency occurs.

From (27) we also obtain the uniqueness in the inverse Neumann problem with three different incident directions if all incident angles are either nonnegative or non-positive.

Finally, we remark that we excluded in our theorem the standard nonuniqueness examples where the two profiles are parallels to the $x_{1}$ axis. In that case it is easy to find counterexamples to the uniqueness in the inverse Dirichlet and Neumann problems for an arbitrarily large number of incident waves.

Acknowledgement. The first author gratefully acknowledges the support by the Department of Mathematical Sciences of the University of Tokyo. The paper was completed while the second author was visiting the Weierstrass Institute of Applied Analysis and Stochastics, and he thanks the institute for the kind invitation. His research was also partly supported by Grant 15340027 from the Japan Society for the Promotion of Science and Grant 15654015 from the Ministry of Education, Cultures, Sports and Technology.

\section{References}

[1] Alessandrini, G. and Rondi, L., Determining a sound-soft polyhedral scatterer by a single far-field measurement. Proc. Amer. Math. Soc. 133 (2005), $1685-1691$.

[2] Bao, G., A uniqueness theorem for an inverse problem in periodic diffractive optics. Inverse Problems 10 (1994), 335 - 340.

[3] Bao, G., Cowsar, L. and Masters, W. (eds.): Mathematical Modeling in Optical Science. Philadelphia: SIAM 2001.

[4] Bebendorf, M., A note on the Poincaré inequality for convex domains. Z. Anal. Anwendungen 22 (2003), 751 - 756.

[5] Bonnet-Bendhia, A.-S. and Starling, F., Guided waves by electromagnetic gratings and non-uniqueness examples for the diffraction problem. Math. Methods Appl. Sci. 17 (1994), 305 - 338. 
[6] Cheng, J. and Yamamoto, M., Uniqueness in an inverse scattering problem within non-trapping polygonal obstacles with at most two incoming waves. Inverse Problems 19 (2003), 1361 - 1384.

[7] Elschner, J. and Schmidt, G., Diffraction in periodic structures and optimal design of binary gratings I. Direct problems and gradient formulas. Math. Methods Appl. Sci. 21 (1998), 1297 - 1342.

[8] Elschner, J. and Yamamoto, M., An inverse problem in periodic diffractive optics: reconstruction of Lipschitz grating profiles. Appl. Anal. 81 (2002), $1307-1328$.

[9] Elschner, J. and Yamamoto, M., Uniqueness in determining polygonal soundhard obstacles with a single incident wave. Inverse Problems 22 (2006), $355-364$.

[10] Elschner, J., Schmidt, G. and Yamamoto, M., An inverse problem in periodic diffractive optics: global uniqueness with a single wavenumber. Inverse Problems 19 (2003), 779 - 787.

[11] Elschner, J., Schmidt, G. and Yamamoto, M., Global uniqueness in determining rectangular periodic structures by scattering data with a single wave number. J. Inverse Ill-Posed Probl. 11 (2003), 235 - 244.

[12] Gilbarg, D. and Trudinger, N. S., Elliptic Partial Differential Equations of Second Order. New York: Springer 1983.

[13] Gotlib, V. Yu., Solutions of the Helmholtz equation, concentrated near a plane periodic boundary. J. Math. Sci. 102 (2000), $4188-4195$.

[14] Hettlich, F. and Kirsch, A., Schiffer's theorem in inverse scattering for periodic structures. Inverse Problems 13 (1997), 351 - 361.

[15] Kamotskiŭ, I. V. and Nazarov, S. A., An augmented scattering matrix and exponentially decreasing solutions of an elliptic problem in a cylindrical domain. J. Math. Sci. 111 (2002), $3657-3666$.

[16] Kirsch, A., Diffraction by periodic structures. In: Proc. Lapland Conf. Inverse Problems (eds.: L. Päivärinta et al). Berlin: Springer 1993, pp. 87 - 102.

[17] Payne, L. E. and Weinberger, H. F., An optimal Poincaré inequality for convex domains. Arch. Rational Mech. Anal. 5 (1960), 286 - 292.

[18] Petit, R. (ed.), Electromagnetic Theory of Gratings. Berlin: Springer 1980.

Received March 29, 2005 